\title{
Superconducting accelerating structures for very low velocity ion beams
}

\author{
J. Xu, ${ }^{*}$ K. W. Shepard, P. N. Ostroumov, J. D. Fuerst, and G. Waldschmidt \\ Argonne National Laboratory, 9700 S. Cass Avenue, Argonne, Illinois 60439, USA \\ I. V. Gonin \\ Fermi National Laboratory, P.O. Box 500, Batavia, Illinois 60510, USA
}

(Received 10 December 2007; published 13 March 2008)

\begin{abstract}
This paper presents designs for four types of very-low-velocity superconducting (SC) accelerating cavity capable of providing several MV of accelerating potential per cavity, and suitable for particle velocities in the range $0.006<v / c<0.06$. Superconducting TEM-class cavities have been widely applied to cw acceleration of ion beams. SC linacs can be formed as an array of independently phased cavities, enabling a variable velocity profile to maximize the output energy for each of a number of different ion species. Several laboratories in the U.S. and Europe are planning exotic beam facilities based on SC linacs. The cavity designs presented here are intended for the front end of such linacs, particularly for the postacceleration of rare isotopes of low charge state. Several types of SC cavities have been developed recently to cover particle velocities above $0.06 \mathrm{c}$. Superconducting four-gap quarter-wave resonators for velocities $0.008<\beta=v / c<0.05$ were developed about two decades ago and have been successfully operated at the ATLAS SC linac at Argonne National Laboratory. Since that time, progress in simulation tools, cavity fabrication, and processing have increased SC cavity gradients by a factor of 3-4. This paper applies these tools to optimize the design of a four-gap quarter-wave resonator for exotic beam facilities and other low-velocity applications.
\end{abstract}

DOI: 10.1103/PhysRevSTAB.11.032001

\section{INTRODUCTION}

The most efficient production mechanisms for slow radioactive ions yield ions in a $1+$ or a $2+$ charge state [1]. In any future exotic beam facility (EBF), a postaccelerator must, to ensure maximum efficiency, be able to accept such low charge-to-mass ratio ions. Very efficient, low-charge-to-mass-ratio injectors have been proposed for this application [2-4]. This type of injector is based on a low-frequency, cw radio frequency quadrupole (RFQ) injecting a very-low-velocity superconducting (SC) linear accelerator. The latter must be capable to accept and accelerate rare isotopes with charge-to-mass ratio in the range from $1 / 238$ to $1 / 6$. The $\mathrm{SC}$ linac is required after the gas stripping for $q / A \geq 1 / 66$ ions in the energy range from $75 \mathrm{keV} / u$ to $\sim 1 \mathrm{MeV} / u$.

Superconducting four-gap quarter-wave resonators, also known as "fork" or interdigital cavities, are a proven type of SC cavity for application in the energy range from $\sim 30 \mathrm{keV} / u$ to $\sim 1.0 \mathrm{MeV} / u$ [2]. At very low velocities, the greater length of 4-gap cavities compared with 2-gap cavities provides more voltage per cavity, while at the same time the number of gaps remains small enough to provide a wide velocity acceptance, critical in an ion linac designed to accelerate a variety of ion species. Four-gap quarterwave resonators (QWR) have been used since 1984 in the ATLAS Positive Ion Injector, and presently operate at accelerating gradients of 3-4 MV/m [5]. Current state-

\footnotetext{
${ }^{*}$ Corresponding author. jin_xu@anl.gov
}

PACS numbers: 85.25.Am, 29.27.-a, 41.75.-i

of-the-art for TEM-class SC cavities, however, provides substantially higher gradients [6,7]. For several types of quarter-wave, half-wave, and spoke-loaded resonators designed for the velocity range $0.1 \mathrm{c}$ to $0.7 \mathrm{c}$, residual $\mathrm{RF}$ surface resistances as low as $R_{S} \approx 5 \mathrm{n} \Omega$ and peak surface electric fields above $30 \mathrm{MV} / \mathrm{m}$ have been reproducibly attained. For the cavities discussed here, such performance corresponds to accelerating gradients above $10 \mathrm{MV} / \mathrm{m}$. The fork cavities combined with SC solenoid or quadrupole focusing can provide $\sim 4 \mathrm{MV} / \mathrm{m}$ real-estate accelerating gradients which are higher than accelerating gradients achievable with SC RFQ structures. For example, a recently commissioned SC RFQ [8] can provide from 1.9 to $2.8 \mathrm{MV} / \mathrm{m}$ accelerating gradients in the energy range from 37 to $540 \mathrm{keV} / u$. An advantage of fork cavities compared to RFQ structures is a capability for rephasing to change the velocity profile and for the acceleration of various ion species to higher velocities.

In what follows, we present designs for several types of four-gap quarter-wave resonant cavity optimized for present state-of-the-art superconducting materials and processing techniques. In Sec. II we establish cavity design goals by briefly discussing the design of a postaccelerator linac. In Sec. III, we detail the optimization of the electromagnetic design and in Sec. IV, the mechanical design. In Sec. V we discuss the results and conclusions.

\section{POSTACCELERATOR FOR RADIOACTIVE ION BEAMS}

As we proposed in previous publications [2-4], a postaccelerator for radioactive ion beams (RIB) includes three 


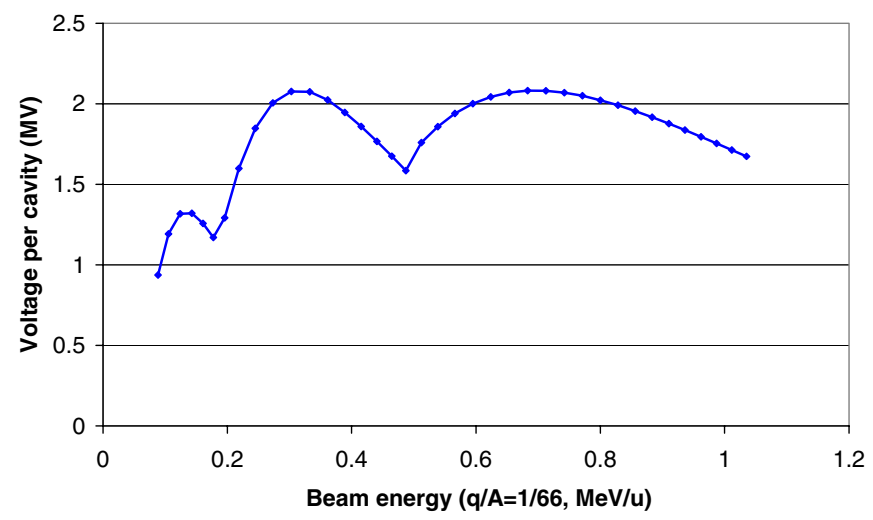

FIG. 1. (Color) Voltage gain per cavity as a function of ion beam energy, $q / A=1 / 66$, Injection energy is $75 \mathrm{keV} / u$.

main sections: a normally conducting (NC) injector capable to accept ions with the lowest charge-to-mass ratio $q / A=1 / 238$ and accelerate up to $\sim 75 \mathrm{keV} / u$, a superconducting (SC) linac for ions with charge-to-mass ratio $1 / 66$ or higher up to an energy of $\sim 1 \mathrm{MeV} / u$, and a higher energy SC linac including existing ATLAS to produce $10 \mathrm{MeV} / u$ beams up to uranium. The main purpose of a NC section comprised of three RFQs is to form lowlongitudinal-emittance beams at bunching frequency of 12.125 MHz with an output energy of $75 \mathrm{keV} / u$. Between the RFQ sections a gas stripper increases the charge state of the heaviest ions to $q / A \geq 1 / 66$ prior to the injection into the SC section. The SC section $\mathrm{cw}$ accelerates the low-charge-state heavy ions to $1 \mathrm{MeV} / u$, requiring a total voltage $\sim 70 \mathrm{MV}$.

We propose to base the $\mathrm{SC}$ section of injector linac on an updated and optimized design of already proven interdigital drift-tube SC niobium cavities. As we show below, such optimized cavities can provide typically $\sim 2 \mathrm{MV}$ of accelerating potential per cavity in this velocity range.

Very-low-charge-state beams, however, require stronger transverse focusing $[2,4]$, particularly at low velocities, than is used in existing SC ion linacs. For the charge states considered here $(q / A=1 / 66)$, the proper focusing can be reached by the help of SC quadrupoles. The linac FODO lattice will consist of a SC quadrupole following each superconducting four-gap cavity. The FODO lattice is tuned to provide phase advance close to $90^{\circ}$ per period to mitigate coupling transverse and longitudinal motions and avoid noticeable growth of longitudinal emittance as discussed in $[2,4]$. Three types of four-gap QWRs operat- ing (conservatively) at $8 \mathrm{MV} / \mathrm{m}$ accelerating field can form the required linac as an array of 38 cavities operating at $-20^{\circ}$ synchronous phase, with the voltage gain per cavity along the linac shown in Fig. 1. Such an array would form a very compact and cost-effective RIB injector, which would require just four cryostat vessels.

Table I lists the basic parameters of four different types of four-gap quarter-wave resonators (four-gap QWR). Type I, type II, and type III are intended for use in a low charge-to-mass ratio $(q / A \geq 1 / 66)$ postaccelerator. The type-IV cavity is intended for a multi-ion, multiplecharge-state driver linac for the EBF.

\section{ELECTROMAGNETIC DESIGN OPTIMIZATION}

To achieve $8-10 \mathrm{MV} / \mathrm{m}$ of accelerating gradient in the proposed 4-gap resonators, the following criteria were chosen: (i) Apply the fabrication technology of SC TEM cavities as developed at ANL for the construction of QWRs. A further design constraint was to minimize prototyping costs for the new four-gap designs by using, to the extent possible, dies previously constructed for 2-gap QWR cavities. (ii) Minimize the ratio of the peak surface electric field to the accelerating field $E_{\mathrm{PEAK}} / E_{\mathrm{ACC}}$. (iii) Minimize the peak magnetic field. (iv) Maximize the cavity geometrical factor and shunt impedance.

The code CST MWS 2006B [9] was used as the main electromagnetic design tool. This version provides more accurate eigenfrequencies and surface fields for resonators with complex geometries than previous versions. The fast perfect boundary approximation (FPBA) available in MWS enabled us to use imported cavity geometries built by the PROE software package [10].

For comparison and a double check on results, another software package, ANALYST, from Simulation Technology and Applied Research, Inc. [11], was also used. Generally, MWS simulations are faster than ANALYST for a similar number of mesh points. However, ANALYST can achieve higher speeds by running on 32 processors.

\section{A. Optimization of cavity parameters}

Current state-of-the-art cavities are fabricated from ( $R R R \geq 250$ ) bulk niobium, in the form of $\sim 3 \mathrm{~mm}$ thick sheets. Die forming and EB welding can produce nearly any desired geometry. A helium vessel is formed between an $\mathrm{Nb}$ cavity and a stainless steel (SS) jacket [12].

TABLE I. Basic parameters of 4-gap QW resonators for the EBF at ANL.

\begin{tabular}{lcccc}
\hline \hline \multicolumn{1}{c}{ Parameter } & Type I & Type II & Type III & Type IV \\
\hline Frequency (MHz) & 48.5 & 48.5 & 72.75 & 57.5 \\
$\beta_{\mathrm{G}}$ & 0.017 & 0.026 & 0.038 & 0.031 \\
Aperture diameter (cm) & 2.0 & 2.0 & 2.0 & 3.0 \\
Number of cavities required for the advanced exotic beam linac & 6 & 12 & 20 & 3 \\
\hline \hline
\end{tabular}




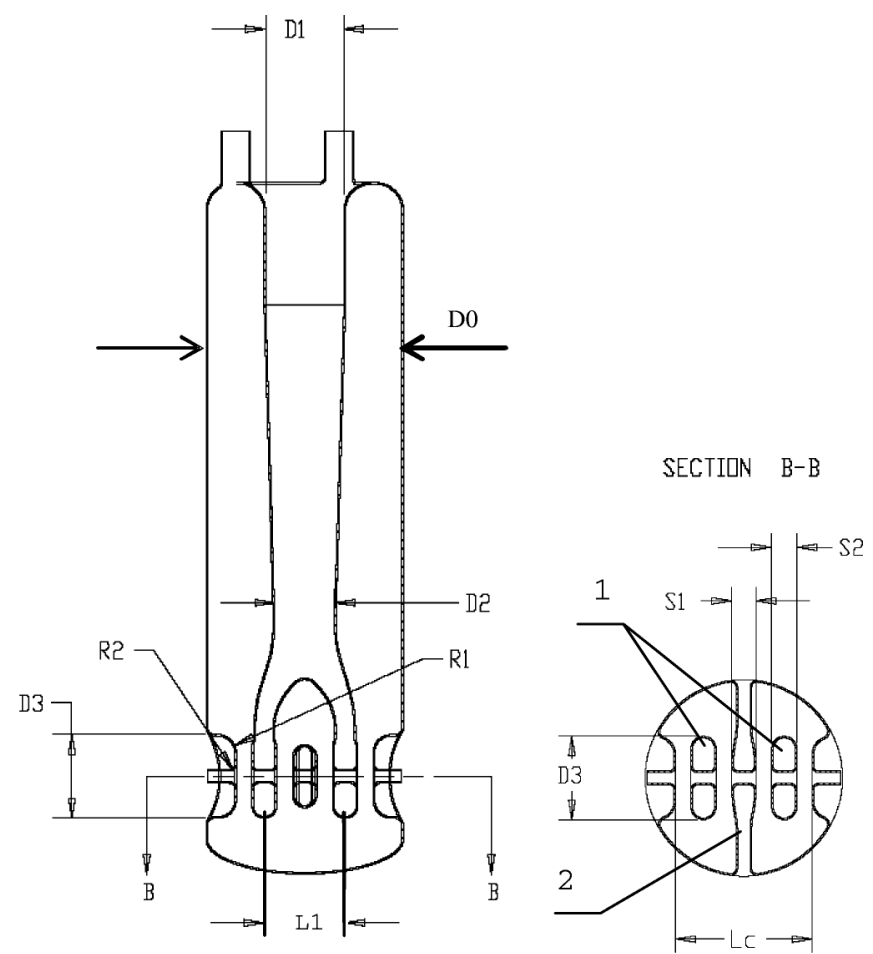

FIG. 2. Main parameters of the cavity to be varied in optimization procedure. 1 -intermediate DTs, 2-horizontal crossbar.

We started with cavity type II (from Table I). A schematic design of the four-gap QWR is shown in Fig. 2. The design of the central drift tube differs from earlier 4-gap cavities, and is now formed as a hollow horizontal bar, allowing direct cooling by liquid helium.

The mechanical stability of a QWR is primarily defined by the length of the central conductor, or stem. The outer diameter of the drift tubes and the stem diameter define the length of the stem and the overall cavity height required for a given frequency.

$E_{\mathrm{PEAK}}$ and $B_{\mathrm{PEAK}}$ have been minimized by varying the main cavity parameters shown in Fig. 2 . To reduce prototype fabrication cost by using the existing dies, the diameter of the cavity $D 0$ and stem $D 1$ have been chosen as for previously built QWRs [7].

First, the ratio $E_{\mathrm{PEAK}} / E_{\mathrm{ACC}}$ was minimized by the following procedures (shown in Fig. 2): (1) Diameter D3 of the outer drift tubes (DT) at the entrance and exit of the cavity has been adjusted. (2) Thickness of DTs and distance L1 between two suspended drift tubes had been changed. These were varied while maintaining design $\beta_{G}$ unchanged as shown in Table I. Intermediate DTs are attached to the stem through the fork therefore their thickness (S2) also affects peak magnetic field. During the optimization procedure, we have tried to apply different shapes of the cross section of the intermediate DTs in the horizontal plane. Instead of the "racetrack" shape as is shown in Fig. 2, a trapezoid shape has been also applied. However, the latter reduces the blending radius and results in a higher peak surface electric field. (3) The blending radius $R 1$ is maximized to be equal to the half of the drifttube thickness. (4) The shape of the horizontal cross-bar which forms the central DT has been optimized. The final shape of the bar is shown in Fig. 2. Ramping of the bar cross section along the horizontal axis reduces peak electric surface field. (5) The blending radius $R 2$ is optimized.

Second, the $B_{\text {PEAK }}$ was minimized by the following procedures (shown in Fig. 2): (1) In early trials, the loft connection between the stem and intermediate drift tubes had a cylindrical cross section. The smallest cylinder radius occurs on the drift-tube surface and equal to the half of the drift-tube thickness $S 2$. The latter is optimized to obtain the lowest $E_{\text {PEAK}}$. The cylinder shape was changed to a racetrack shape, which significantly reduced local $B_{\mathrm{PEAK}}$. (2) The diameter D2 of the stem cone (see Fig. 2) was optimized. A conical shape for the stems has been chosen to minimize multipacting, in accord with previous experience with QWR cavities.

The above optimization procedure was iteratively repeated to minimize $E_{\mathrm{PEAK}}$ and $B_{\mathrm{PEAK}}$, while keeping the cavity geometrical factor $G$ and the ratio of shunt impedance to quality factor $R / Q_{0}$ high.

The shapes of cavity types I, III, and IV were obtained by modifications of the type-II geometry applying the following procedures.

Type I. - The geometry has been scaled in all dimensions by a factor. The cavity (and stem) height were adjusted to arrive with the correct frequency.

Type III. - A higher velocity was obtained by increasing the frequency by reducing the stem length to obtain a frequency of $72.75 \mathrm{MHz}$, the 6th harmonic of the bunching frequency, while the drift tubes and fork section of the cavity were kept the same as for the type-II cavity.

Type IV. - The distance between the intermediate drift tubes $L 1$ and cavity length $L_{c}$ has been increased to
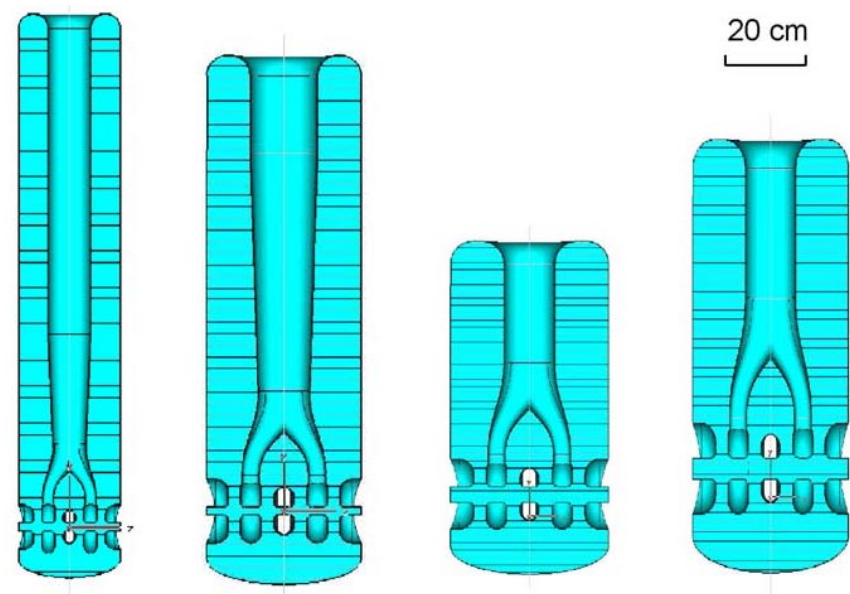

Type-I

Type-II

Type-III

Type-IV

FIG. 3. (Color) Mws models of four types of 4-gap quarter-wave resonators. 
TABLE II. Main electrodynamics parameters of four-gap QWRs given for $E_{\mathrm{ACC}}=1 \mathrm{MV} / \mathrm{m}$.

\begin{tabular}{lcccccc}
\hline \hline Parameter & Units & ATLAS & Type I & Type II & Type III & Type IV \\
\hline$E_{\text {PEAK }}$ & $\mathrm{MV} / \mathrm{m}$ & 5.04 & 3.76 & 3.41 & 3.48 & 3.61 \\
$B_{\text {PEAK }}$ & Gauss & 117 & 42 & 43 & 55 & 48 \\
Length, $L_{c}$ & $\mathrm{~cm}$ & 24.638 & 17 & 26 & 26 & 26.4 \\
$W$ & $\mathrm{~mJ}$ & 221 & 72 & 150 & 118 & 149 \\
$G$ & $\Omega$ & 13.8 & 11.5 & 18.1 & 25.1 & 20.9 \\
$R / Q_{0}$ & $\Omega$ & 900 & 1309 & 1486 & 1254 & 1298 \\
$\beta_{\mathrm{G}}$ & & 0.025 & 0.017 & 0.026 & 0.038 & 0.031 \\
Height & $\mathrm{cm}$ & 110.5 & 139.5 & 131.3 & 80.8 & 107.3 \\
Diameter & $\mathrm{cm}$ & 30.48 & 23.54 & 37.0 & 37.0 & 37.0 \\
\hline \hline
\end{tabular}

provide slightly higher $\beta_{G}$ and adjusting the cavity height to obtain $57.5 \mathrm{MHz}$ resonant frequency.

The cross sections of all four MWs models in the $(y, z)$ plane are shown in Fig. 3. The optimized electromagnetic parameters of all cavity types are summarized in Table II. For the comparison, we also list parameters of an earlier four-gap $48.5 \mathrm{MHz}$ cavity design presently used in the existing ATLAS SC linac. In the optimized cavities, both the peak electric and magnetic field are substantially lower than for ATLAS cavity.

Figure 4 shows, for the type-II cavity, the first trial and the final geometry of the cavity, together with the distribution of the surface electric field.

\section{B. Comparison of MWS and ANALYST results}

Peak surface fields in a resonant cavity of complex shape are quite sensitive to the parameters of computer model and simulation technique. Two commercial codes, MWS and ANALYST, have different algorithms to solve Maxwell equations. We have applied the latest versions of both codes with different mesh sizes to compare the simulation results of type-II cavity. The results are summarized in Table III. It can be seen that the ANALYST results obtained on $1.6 \times 10^{6}$ mesh elements converged to the MWS results. Points A and B indicate locations of the peak surface electric field. Even with a relatively large number of mesh points, MWs produces isolated regions with suspiciously and anomalously high peak surface fields while ANALYST achieves a more continuous approximation of the surface fields with a similar number of mesh points. The computation speed of MWS is much faster than ANALYST: therefore we used the parallel version of ANALYST running on the ANL BG/L supercomputer. Using the parallel version of ANALYST was important for confirming the MWS
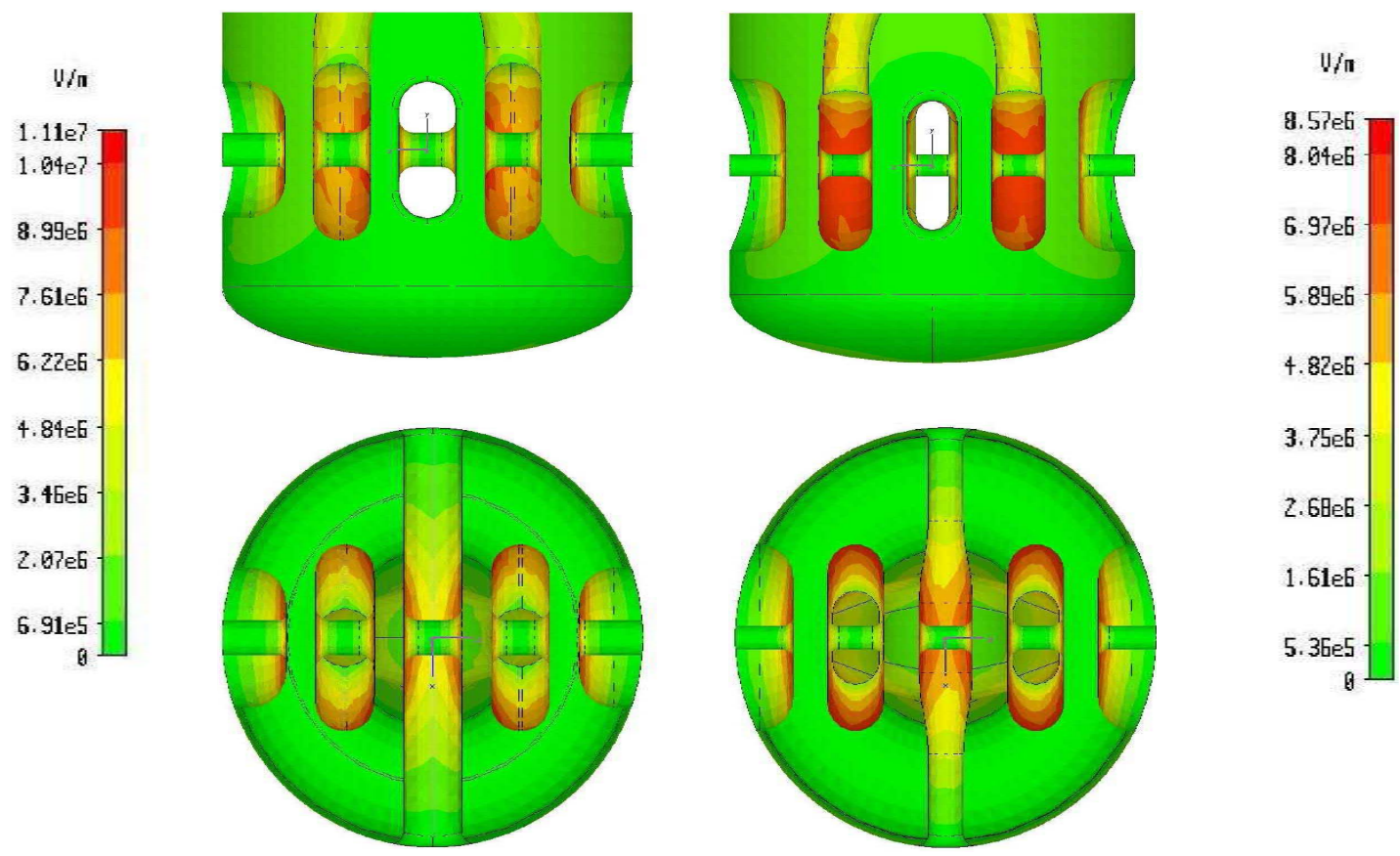

FIG. 4. (Color) Surface electric field distribution in $(x, z)$ and $(y, z)$ planes. The preliminary design (on the left) and optimized design (on the right). 
TABLE III. Comparisons of type-II cavity simulations performed with MWS and ANALYST. All data are given for $E_{\mathrm{ACC}}=1 \mathrm{MV} / \mathrm{m}$.

\begin{tabular}{lccccc}
\hline \hline Parameter & Units & \multicolumn{2}{c}{ MWS (PBA mesh) } & \multicolumn{2}{c}{ ANALYST } \\
\hline Mesh points & & $390 \mathrm{~K}$ & $2 \mathrm{M}$ & $68 \mathrm{~K}(\mathrm{~h} 1.5)$ & $1.6 \mathrm{M}(\mathrm{h} 1.5)$ \\
$E_{\text {PEAK }}$ & $\mathrm{MV} / \mathrm{m}$ & 3.48 & 3.41 & 4.12 & 3.47 \\
$B_{\text {PEAK }}$ & Gauss & 40.2 & 40.5 & 65 & 42.3 \\
$G$ & $\Omega$ & 17.7 & 18.1 & 18.1 & 16.9 \\
$R / Q_{0}$ & $\Omega$ & 1473 & 1486 & 1330 & 1463 \\
$f_{0}$ & $\mathrm{MHz}$ & 48.66 & 48.41 & 48.88 & 48.52 \\
\hline \hline
\end{tabular}

simulations. Figure 5 shows the electric field contours in the $(y, z)$ plane obtained by both MWS and ANALYST codes. It may be noted that the field approximation near the rounded surfaces is more accurate in ANALYST code which
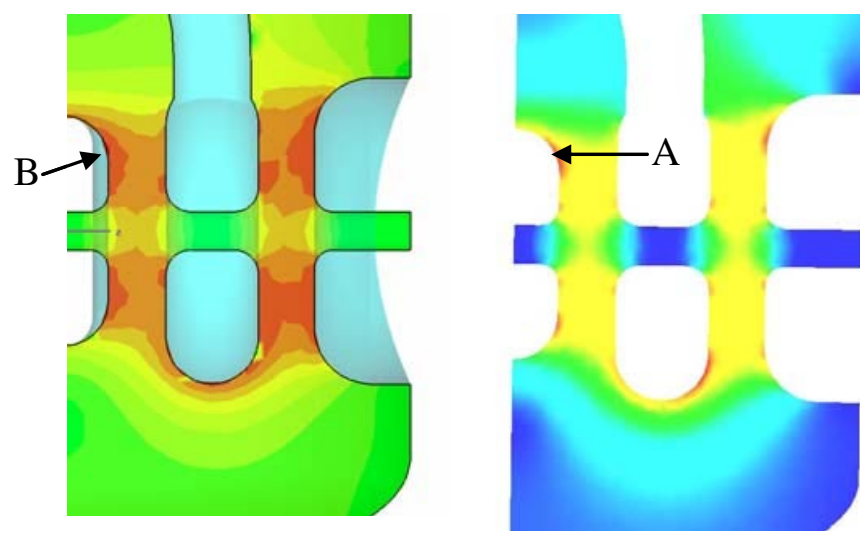

FIG. 5. (Color) Electric field contours in the $(y, z)$ plane simulated with MWS with $2 \times 10^{6}$ mesh points (on the left) and ANALYST with $1.6 \times 10^{6}$ mesh elements (on the right).

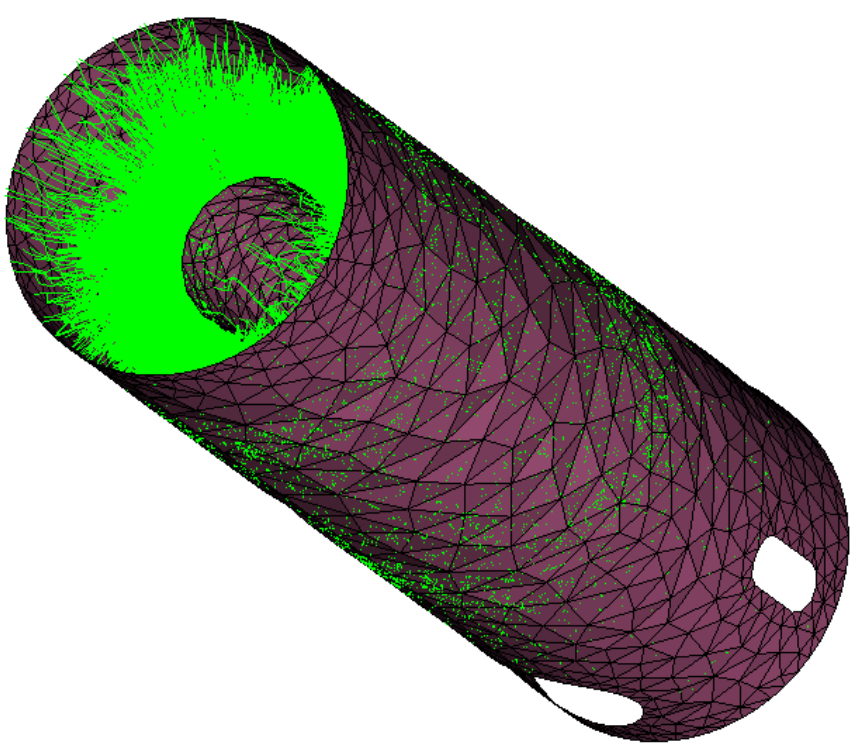

FIG. 6. (Color) Electron trajectories between surfaces of the stem and housing cylinder. uses the finite element method. The ANALYST software is also more adequate for the study of multipacting phenomena in resonators and was applied to identify multipacting in the space between the stem and housing cylinder. This region is of particular interest due to its coaxial shape. In our multipacting simulation, we use enhanced counter function $\left[e_{n}(|F|)=\sum_{j=1}^{N_{0}} N_{n}\left(x_{0}^{j}\right)\right]$ to describe the total number of electrons, including original and the secondary electrons emitted from the wall due to the impacts. $x_{0}^{j}$ is the initial position for the $j$ th electron. The total number of secondary electrons due to a single electron launched after $n$ impacts is given by $N_{n}\left(p_{0}\right)=$ $\prod_{k=1}^{n} \delta\left(x_{k}, E_{k}\right)$, where $\delta\left(x_{k}, E_{k}\right)$ is the ratio of total electrons emitted from the wall to the total number of electrons bombarding the wall at location $x_{k}$ and at energy level $E_{k}$. Figure 6 shows the electron trajectories after 10 impacts between the surfaces of the stem and housing cylinder. Figure 7 shows enhanced counter functions after 5, 10, and 20 impacts. It is clear that enhanced counter function diminished as the number of impacts grows. This figure indicates there are no multipacting activities over the stem area.

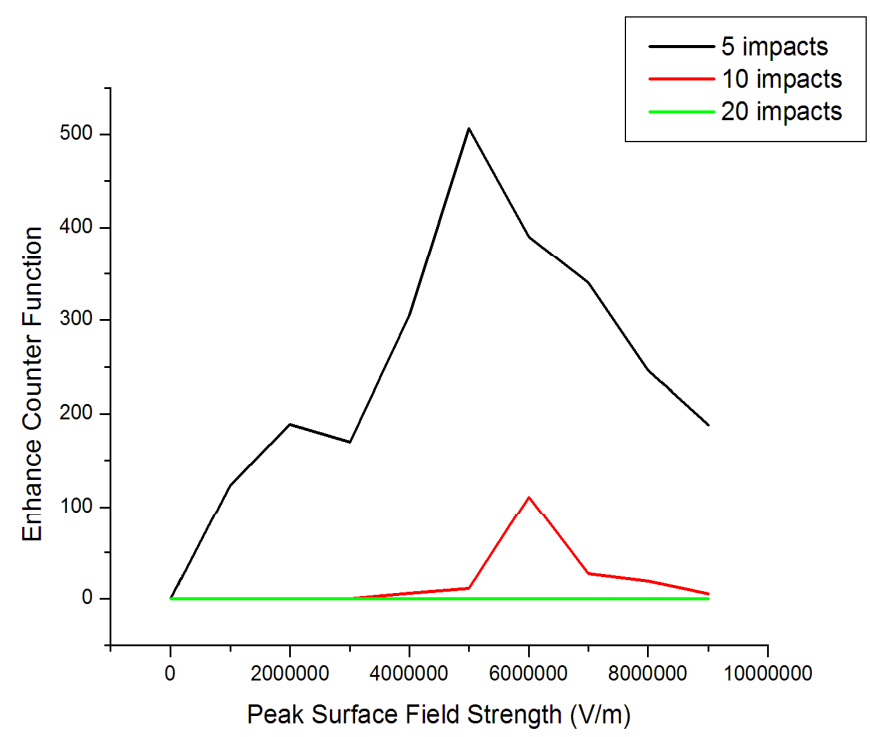

FIG. 7. (Color) Enhance counter functions after 5, 10, and 20 impacts. 


\section{STRUCTURAL ANALYSIS}

The frequency and phase stability of high- $Q$ SC cavities are extremely sensitive to mechanical deformation [13]. For $\mathrm{cw}$ applications, the dominant driver of mechanical deformations is microphonic vibrations due to various external sources. In QWRs, ambient microphonic noise can also excite low-frequency mechanical resonances of the cavity [14]. One source of microphonics in operation at 4.2 $\mathrm{K}$ is that RF losses cause appreciable boiling in the liquid helium, resulting in small fluctuations in the local helium pressure [15]. The reduction of the cavity sensitivity to microphonic noise is crucial in $\mathrm{cw}$ low-current operation of SC cavities when beam loading is negligible.

Several techniques have been developed to control the cavity RF phase in the presence of microphonic noise [13]. Effective damping techniques have been proposed and implemented to damp the mechanical resonance using passive absorbers of energy of mechanical oscillations [14]. Structural strengthening of the cavity during the mechanical design to minimize the resonant frequency sensitivity to the helium pressure is an effective measure to control microphonics [15].

As part of the design process, we performed detailed mechanical modeling of the type-II cavity. A detailed PROE model of the type-II cavity is shown in Fig. 8. We have performed simulations of the cavity with two symmetry planes using ANSYS multiphysics [16] software package to study the effects of (a) a frequency shift due to the helium pressure and (b) a frequency response to an external slowtuning device. The structural analysis of the cavity shown in Fig. 9 under $10^{5} \mathrm{~Pa}$ helium pressure has been performed using both PROE and ANSYS software packages. Both software packages show significant displacement of the stem, $\sim 0.5 \mathrm{~mm}$, in the vertical direction. As a result the frequency shift can reach to $-27 \mathrm{kHz}$, which was unaccept-

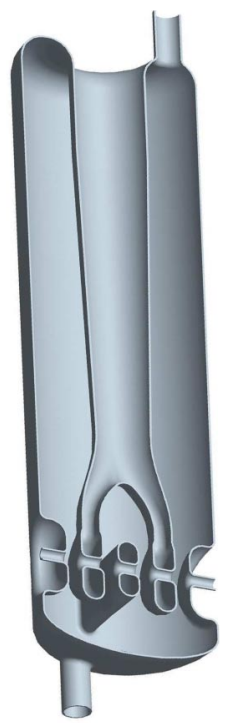

FIG. 8. (Color) PROE model of the type-II cavity.

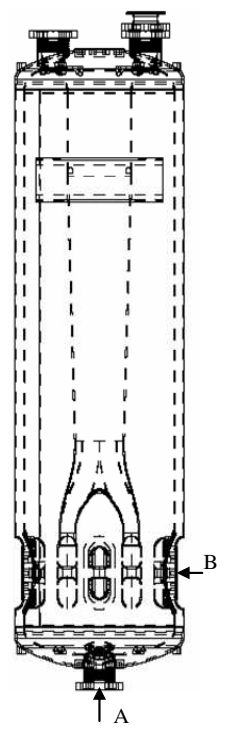

FIG. 9. Cross section of the type-II cavity in the SS jacket.

ably large. To reduce this frequency shift, the cavity design was modified by structurally enhancing the connection of the stem to the cylinder, and by stiffening the horizontal cross-bar. To find the effect of helium pressure on the cavity frequency, two cases were studied: (1) the cavity fixed at one point (point A in Fig. 9) and (2) the cavity fixed at the beampipe surface, surface B (see Fig. 9). The ANSYS results are shown in Fig. 10 and summarized in Table IV.
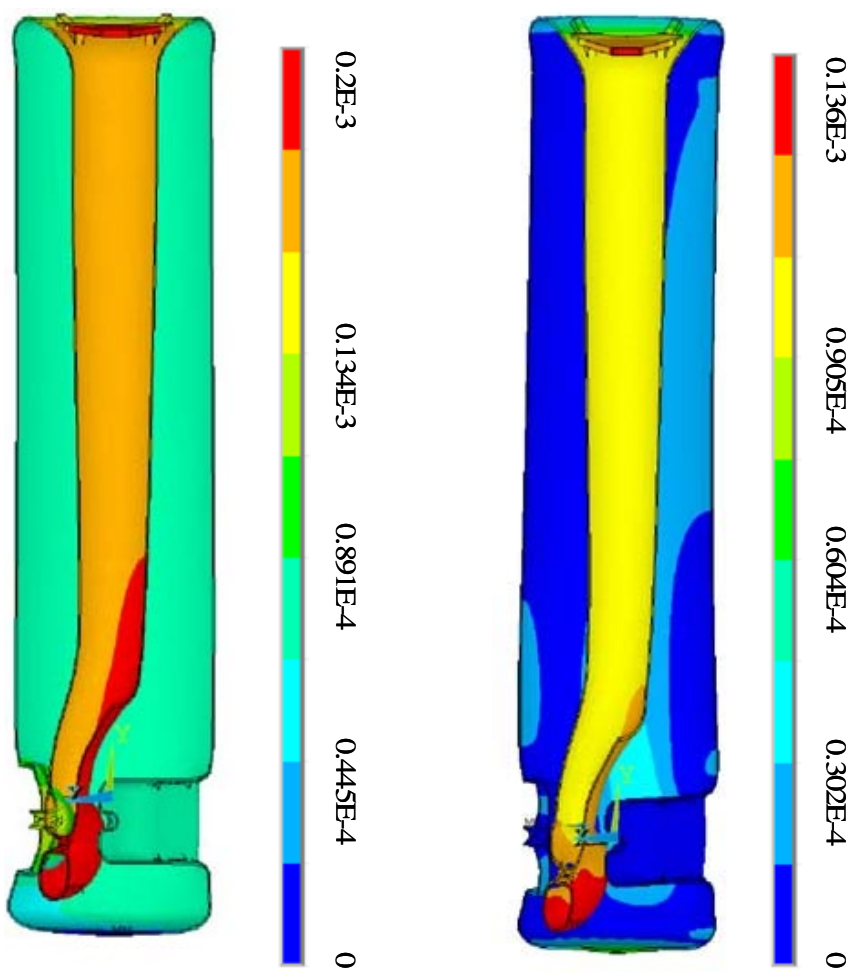

FIG. 10. (Color) ANSYS results of deformed geometries for case 1 (left) and case 2 (right). Only $\frac{1}{4}$ of the cavity is shown. 
TABLE IV. ANSYS simulation results for frequency detuning due to $10^{5} \mathrm{~Pa}$ helium pressure.

\begin{tabular}{lc}
\hline \hline Mode number & 1 (operational) \\
Initial frequency $(\mathrm{MHz})$ & 48.297 \\
Frequency shift, case 1 (kHz) & -13 \\
Frequency shift, case 2 (kHz) & -5 \\
\hline \hline
\end{tabular}

The maximum displacement of the cavity walls is $200 \mu \mathrm{m}$ and $136 \mu \mathrm{m}$ for the first and second cases, respectively. As is seen from Table IV, the frequency shift is between $-13 \mathrm{kHz}$ to $-5 \mathrm{kHz}$ for the two cases being studied. During the mechanical design of the cavities, the cavity sensitivity to the helium pressure will be further decreased by further stiffening of the stem support. In addition, the stainless steel jacket will also reduce the sensitivity of the cavity resonant frequency to the helium pressure.

A mechanical tuning mechanism will be applied symmetrically from both sides of the cavity to the surface B as shown in Fig. 9. In ANSYS simulations of the tuner effect, the cavity is fixed at the bottom point A (Fig. 9). A pulling force of $1120 \mathrm{~N}$ was applied over the $26 \mathrm{~mm}$ diameter of the aperture pipe. The deformed geometries of the cavity are shown in Fig. 11, and frequency shifts are listed in Table V. Sensitivity of the cavity frequency to the tuner force is $\sim 12.5 \mathrm{~Hz} / \mathrm{N}$. As can be seen from Tables IV and V, the applied force approximately compensates frequency shift due to the helium pressure.
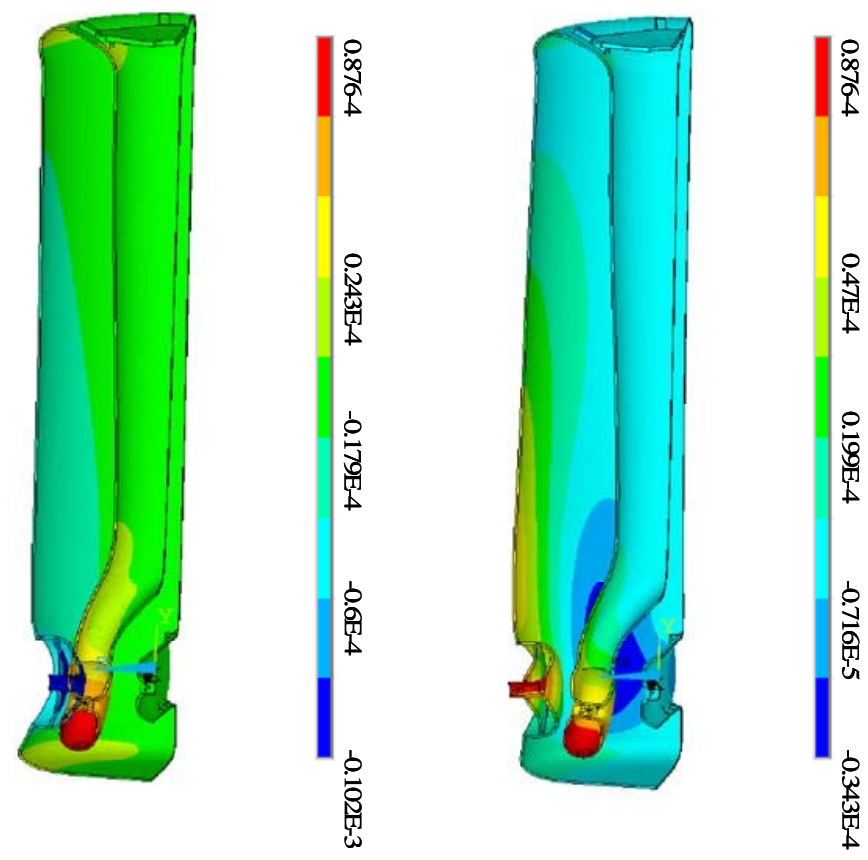

FIG. 11. (Color) ANSYS results of the cavity deformations in the $Z$ direction along the beam line due to the helium pressure (left) and the combined effect of the helium pressure and mechanical tuner with pulling force (right). Only $\frac{1}{4}$ of the cavity is shown.
TABLE V. ANSYS simulation results for frequency detuning due to the effect of the tuner.

\begin{tabular}{lc}
\hline Mode number & 1 (operational) \\
Initial frequency $(\mathrm{MHz})$ & 48.297 \\
Frequency shift, pressure is applied $(\mathrm{kHz})$ & 1 \\
Frequency shift, no pressure $(\mathrm{kHz})$ & 15 \\
\hline \hline
\end{tabular}

\section{DISCUSSION AND CONCLUSIONS}

Four-gap interdigital quarter-wave superconducting cavities can provide effective and efficient means of accelerating ion beams in the velocity range from $0.012 \mathrm{c}$ to $0.05 \mathrm{c}$. Earlier versions of these types of cavities were developed, built, and operated in the Argonne heavy-ion linac ATLAS. The ATLAS four-gap QWRs built in the 1980s are operated typically at $\sim 3 \mathrm{MV} / \mathrm{m}$ accelerating field which corresponds to $E_{\mathrm{PEAK}} \approx 15.1 \mathrm{MV} / \mathrm{m}$ and $B_{\mathrm{PEAK}} \approx 360 \mathrm{G}$.

Extensive optimization of four-gap cavity mechanical and electrodynamics properties has been performed using the most recent versions of modern three-dimensional software tools. As a result of these studies, peak magnetic and electric surface fields have been significantly reduced to $E_{\mathrm{PEAK}} \approx 3.4 \mathrm{MV} / \mathrm{m}$ and $B_{\mathrm{PEAK}} \approx 43 \mathrm{G}$ per $1 \mathrm{MV} / \mathrm{m}$ of the accelerating field. The reduction of peak electric field has been achieved by optimization of the drift tube and horizontal bar shapes. Similarly, the magnetic field has been reduced by optimization of the conical stem and lofts connecting the stem to the drift tubes. With modern fabrication technology and cavity processing techniques, peak surface electric fields can be increased to $\sim 32-35 \mathrm{MV} / \mathrm{m}$ which have been demonstrated in numerous prototype TEM-class cavities. Therefore optimally designed fourgap cavities, using modern construction and processing techniques, can provide $\sim 10 \mathrm{MV} / \mathrm{m}$ accelerating fields, or more than $2 \mathrm{MV}$ of accelerating voltage per cavity. The accelerating gradients provided by four-gap cavities are higher by the factor 3 and 4 compared to the existing accelerators in the similar velocity range $[8,17]$.

Effective use of 3D design and analysis codes allows us to skip prototyping of all types of cavities. Our intention is to build a prototype cavity of type II that can be later used in the accelerator. The main purpose of cavity prototyping is to resolve engineering issues and to understand the production cost of these cavities.

There are several other issues related to the highperformance cavity design. Reduction of the frequency sensitivity to the variation of the helium pressure is crucial for application in cw low-current accelerators. This parameter has been reduced by the enhancement of the stem connection to the cylindrical body and by strengthening of the horizontal bar which forms the middle drift tube.

High-performance high-gradient four-gap cavities can significantly reduce the number of cavities required to provide $\sim 70 \mathrm{MV}$ accelerating voltage for the low charge state postaccelerator. The optimized design, combined 
with recently developed cavity fabrication and cleaning techniques, will reduce the cost of proposed accelerators for any future EBF project.

\section{ACKNOWLEDGMENTS}

This work was supported by the U.S. Department of Energy, Office of Nuclear Physics and High Energy Physics, under Contracts No. DE-AC-02-06CH11357 and No. DE-AC02-07CH11359.

[1] J. A. Nolen, Nucl. Phys. A734, 661 (2004).

[2] J. W. Kim, K. W. Shepard, and J. A. Nolen, Proceedings of the PAC-1995, Dallas (IEEE, Dallas, TX, 1995), p. 1408.

[3] P. N. Ostroumov, K. W. Shepard, A. A. Kolomiets, J. A. Nolen, and R.C. Pardo, Proceedings of the PAC-2001 (IEEE, Chicago, IL, 2001), p. 4080.

[4] P.N. Ostroumov, V.N. Aseev, and A. A. Kolomiets, Proceedings of the PAC-2005 (IEEE, Knoxville, TN, 2005), p. 3301.

[5] K.W. Shepard, Proceedings of the PAC-1985 (IEEE, Vancouver, 1985), p. 3574.

[6] M. P. Kelly, J.D. Fuerst, M. Kedzie, and K. W. Shepard,
Proceedings of the LINAC 2004 (DESY \& GSI, Lübeck, Germany, 2004), p. 608.

[7] M. P. Kelly, Z. A. Conway, J. D. Fuerst, M. Kedzie, and K. W. Shepard, Proceedings of the LINAC 2004 (DESY \& GSI, Lübeck, Germany, 2004), p. 605.

[8] A. Pisent et al., Proceedings of the Linac-2006 (ORNL/ SNS, Knoxville, TN, 2006), p. 227.

[9] CST Microwave Studio, http://www.cst.com/.

[10] http://www.tristar.com/.

[11] http://www.staarinc.com/.

[12] K. W. Shepard, J. D. Fuerst, M. Kedzie, and M. P. Kelly, Proceedings of the PAC-2003 (IEEE, Portland, OR, 2003), p. 1297.

[13] H. Padamsee, J. Knobloch, and T. Hays, $R F$ Superconductivity for Accelerators (Wiley, New York, 1998).

[14] A. Facco and G. Zinkann, Part. Accel. 61, 265 (1998).

[15] Z. A. Conway, K. W. Shepard, M. P. Kelly, J. D. Fuerst, and M. Kedzie, Proceedings of the 2005 SRF Workshop (Cornell University, Ithaca, New York, 2005), http:// www.Ins.cornell.edu/public/SRF2005/Proceedings.html.

[16] http://www.ansys.com/products/multiphysics.asp.

[17] L. M. Bollinger, Proceedings of LINAC '98 Conference, Chicago, IL, 1998 (ANL \& Fermi Lab, Chicago, IL, 1998). 